\title{
Application of finite element methods to photonic crystal modelling
}

\author{
B.P. Hiett, J.M. Generowicz, S.J. Cox, M. Molinari, D.H. Beckett and K.S. Thomas
}

\begin{abstract}
Photonic Crystals (PCs) are materials with a periodically modulated dielectric constant, through which certain frequencies of electromagnetic radiation cannot propagate. The modes admitted by PCs can be investigated effectively using the finite element method with the assistance of the Floquet-Bloch theorem, by considering a unit cell of the material and imposing periodic boundary conditions. Along with the Dirichlet and metric matrices, a third type of elemental matrix emerges. The types of results that are of interest to photonic crystal manufacturers are introduced and presented; in this context, the benefits of using the subspace iteration method to solve the eigensystems are discussed. The performance of the algorithm is investigated with respect to mesh granularity and interpolation order.
\end{abstract}

\section{Introduction}

Photonic band gap (PBG) materials are periodic dielectric crystals that exhibit a PBG analogous to the electronic band gap present in semiconductors. Photons in the frequency range of the band gap are completely excluded so that atoms within such materials are unable to spontaneously absorb and re-emit light in this region [1].

PCs offer enormous potential in the development of highly efficient narrow band (tuneable) lasers, integrated optical computing and high-speed optical communication networks, particularly in the production of purely optical circuits for dense wavelength division multiplexing [2]. An example of a bridge waveguide structure is shown in Fig. 1.

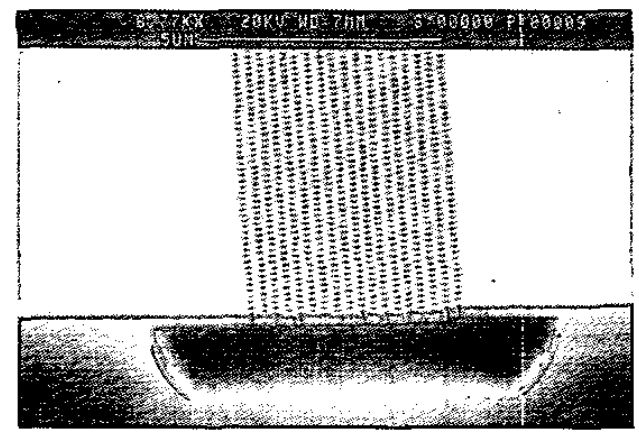

Fig. 1 Bridge waveguide structure (pitch $=300 \mathrm{~nm}$ ) courtesy of Martin Charlton, Southampton microelectronics research group

The electric permittivity of a PBG material varies periodically on a scale comparable to the wavelength of the forbidden photons [3]; hence, the manufacturing of PCs that operate in the visible region of the electromagnetic spectrum requires fabrication techniques with a resolution

\section{(1) IEE. 2002}

IEE Proceedings online no. 20020642

doi:10.1049/ip-smt:20020642

Paper first received 14th March 2002 and in revised form 1 lth June 2002

The authors are with the University of Southampton. Department of Electronics and Computer Science, Southampton SO17 1BJ. UK of approximately $10^{-6} \mathrm{~m}$. These are typically very expensive, therefore an accurate and efficient model is desirable to allow a PC's optical properties to be computed prior to fabrication.

Success in the fabrication of such materials with band gaps in optical frequencies has been limited mainly to those in which the periodicity is restricted to two-dimensions. The in-plane propagation modes of such materials can be investigated effectively using two-dimensional Lagrangian finite elements [4], which yields a considerable reduction in complexity with respect to the three-dimensional curlconforming treatment that would otherwise be necessary.

\section{2D Scalar spectral problem}

In the context of non-magnetic media, Maxwell's equations can be re-expressed as:

$$
\begin{gathered}
\nabla \times \frac{1}{\epsilon(\boldsymbol{x})} \nabla \times \boldsymbol{H}=\left(\frac{\omega}{c}\right)^{2} \boldsymbol{H} \\
\nabla \cdot \boldsymbol{H}=0
\end{gathered}
$$

When the PC structure is invariant in one-dimension Maxwell's equations can be split into two scalar spectral problems dealing with the transverse electric polarisation (TE mode) and the transverse magnetic polarisation (TM mode) respectively:

$$
\begin{gathered}
\text { TE mode : }-\nabla \cdot \frac{1}{\epsilon(\boldsymbol{x})} \nabla \psi=\left(\frac{\omega}{c}\right)^{2} \psi \\
\text { TM mode }: \frac{1}{\epsilon(\boldsymbol{x})} \nabla^{2} \psi=\left(\frac{\omega}{c}\right)^{2} \psi
\end{gathered}
$$

where $\varepsilon(x)$ is the material permittivity at spatial position $\boldsymbol{x}$, $\omega$ is the frequency, $c$ the speed of light and $\psi$ is the scalar field intensity.

\section{$3 \quad$ Modelling periodic structures}

An example of mesh representation of a $\mathrm{PC}$ is shown in Fig. 2. Domain discretisation into finite elements is performed using public domain meshing software (Easymesh by Niceneco [5] and Geompack by Joe [6]) to produce 

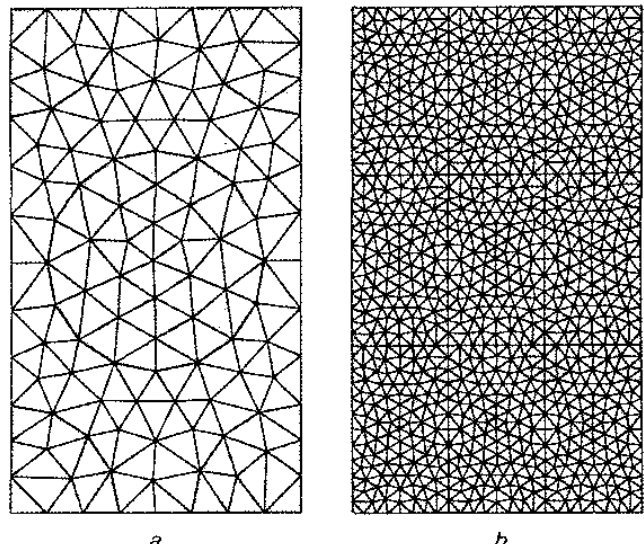

Fig. 2 Unstructured mesh representation of a triangular lattice of rods

$a$ The unit cell used for computation

$b$ The mcsh is periodic with one repeat shown in all directions

a periodic unit cell where the mesh is wrapped such that opposite boundary nodes meet.

The periodic structure of the PC allows the application of the Floquet-Bloch theory in which a crystal of infinite extent can be modelled using a unit cell with periodic boundary conditions [7]. The eigenmodes then take the form:

$$
\psi=e^{i k \cdot x} u(\boldsymbol{x})
$$

where $\boldsymbol{k}$ is the Bloch-quasimomentum vector and $u(\boldsymbol{x})$ is the value of the eigenfunctions at spatial position $x$. The resulting change in the gradient operator:

$$
\nabla \rightarrow \nabla+i k
$$

gives rise to an eigensystem of the form:

$$
\left(\boldsymbol{S}+\boldsymbol{k} \cdot \boldsymbol{P}+\boldsymbol{k}^{2} \boldsymbol{T}\right) \psi=\lambda \boldsymbol{T} \psi
$$

Or,

$$
\boldsymbol{A}(\boldsymbol{k}) \psi=\hat{\boldsymbol{B}} \boldsymbol{B} \psi
$$

where $\boldsymbol{S}$ and $\boldsymbol{T}$ are the familiar Dirichlet and metric matrices:

$$
\begin{gathered}
\boldsymbol{S}=\int_{\Omega} \nabla \alpha_{m} . \nabla \alpha_{n} d \Omega \\
\boldsymbol{T}=\int_{\Omega} \alpha_{m} \alpha_{n} d \Omega
\end{gathered}
$$

$\boldsymbol{P}$ is a vector of matrices assembled from elemental matrices that are defined in terms of the basis functions, $\alpha_{i}$ as:

$$
\boldsymbol{P}_{i j}^{e}=\int \alpha_{i} \nabla \alpha_{j}-\alpha_{j} \nabla \alpha_{i} d \Omega
$$

The elemental matrices are assembled to form the generalised eigenvalue problem which is solved as a function of $\boldsymbol{k}$. For each $\boldsymbol{k}$, the solver computes a set of $i$ eigenvalues. These eigenvalues can be plotted as a function of $\boldsymbol{k}$ to give a dispersion relation or band diagram. The union of band functions for all $\boldsymbol{k}$ represents the density of states for the modes of the original problem. This is essentially a histogram of the mode frequencies. It gives a clear visual indication of any band gaps as the density drops to zero for those frequency ranges. An important feature of the Floquet-Bloch theory is that different $\boldsymbol{k}$ values do not necessarily produce different modes. The vector $\boldsymbol{k}$ and the vector $\boldsymbol{k}+\boldsymbol{G}$, where $\boldsymbol{G}$ is a reciprocal lattice vector produce the same mode. Hence, to avoid solution redundancy one need only consider those values of $\boldsymbol{k}$ that are unique under translational symmetry. This set is said to lie within the first
Brillouin zone [8]. Further mirror and rotational symmetries may allow this set to be reduced even further to produce an irreducible Brillouin zone. The construction of this zone is illustrated in Fig. 3. X, K and $\Gamma$ are commonly used to mark the vertices of the irreducible zone.

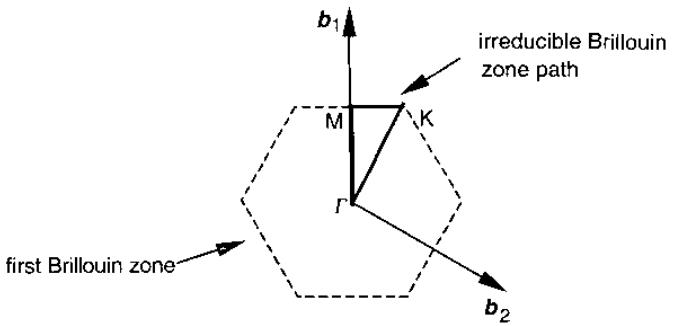

'Fig. 3 Brillouin zone construction in reciprocal lattice space; $\boldsymbol{b}_{l}$ and $\boldsymbol{b}_{2}$ represent the reciprocal lattice vectors.

In practice, the spectrum is approximated by selecting a discrete set of $\boldsymbol{k}$ vectors sampled from the irreducible Brillouin zone. This set of vectors is generated by sampling a number of points from the path describing the reduced Brillouin zone, e.g. the $\mathrm{M}, \Gamma$, X triangle shown in Fig. 3 for a triangular lattice. For more irregular structures in which a reduced Brillouin zone cannot be easily constructed, the set of vectors is chosen using a Sobol sequence [9]. A Sobol sequence, shown in Fig. 4, is a quasi-random selection of points that are distributed evenly across the Brillouin zone and are sorted such that each successive $\boldsymbol{k}$ vector solved is close to the previous one. Both approaches allow optimisations to be made to the solver as discussed in the following section.
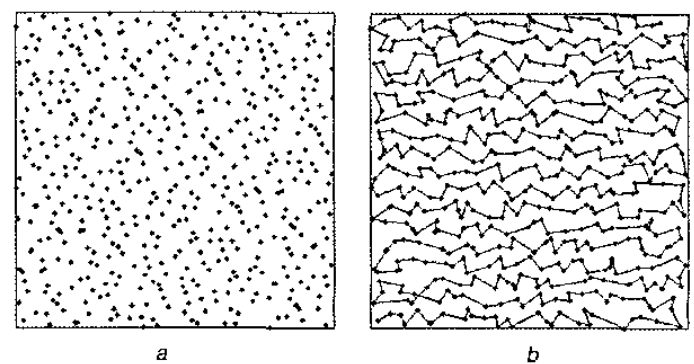

Fig. 4 A Sobol sequence

a A quasi-random distribution of points

$b$ The linking of adjacent points highlighting their close proximity

\section{Subspace iteration}

The analysis of PC structures is aimed at detecting the presence of band gaps. These are generally found between the lowest modes of propagation, therefore, it is only necessary to compute a small number of the lowest eigenstates. The subspace iterative (SI) method [10] suits this application well as it calculates a subset (either the most or least dominant) of the total number of eigenvalues. The method is based on a combination of the power method [11], where several vectors are iterated upon simultaneously, and the properties of the Rayleigh quotient [12]. Conversion to the standard eigenvalue format is not required, so full advantage can be taken of the sparsity and symmetry of the matrices.

Using SI techniques to solve the eigensystem allows several problem specific optimisations to be made. Eigen- 
systems corresponding to similar $\boldsymbol{k}$-vectors differ only slightly, hence using the previous solution as a starting point for the subsequent subspace search significantly reduces the number of iterations required to converge.

The positioning of non-zero matrices is governed solely by the mesh node numbering and is invariant for all $\boldsymbol{k}_{i}$ vectors. Preconditioning of the $\boldsymbol{A}$ matrix (7) and (8) is therefore performed only once using a symmetric approximate minimum degree algorithm [13] to reduce the bandwidth and subsequently improve the performance of the SI solver for all $i$ eigensystems.

In addition, the SI method requires specification of the accuracy to which the eigenvalues must converge. Hence a wide range of $\mathrm{PC}$ structures may be investigated at a low accuracy, those that show promise can then be investigated to a greater convergence tolerance.

\section{Results}

The photonic spectra of many different PC structures have been examined both experimentally, using fabricated devices and lasers [14], and theoretically, using a variety of numerical methods including plane wave expansion techniques and finite difference time domain (FDTD) methods [15].

A commonly modelled and fabricated PC structure is the triangular lattice [16], a photo of a PBG device based on this structure is shown in Fig. 1. Circular rods are arranged in a background material such that their centres are positioned at the lattice points, as illustrated in Fig. 2. Such a structure was modelled in which circular rods of refractive index one i.e. air rods were embedded in a material of dielectric constant 11.4 i.e. gallium arsenide (GaAs). The filling fraction (ratio of rod area to cell area) was $80 \%$.

The finite element method (FEM) analysis of the PC is presented in Fig. 5. A complete PBG (both TE and TM modes) can be observed between a normalised frequency, $\omega a / 2 \pi c$, of 0.44 and 0.48 where $a$ is the lattice constant.

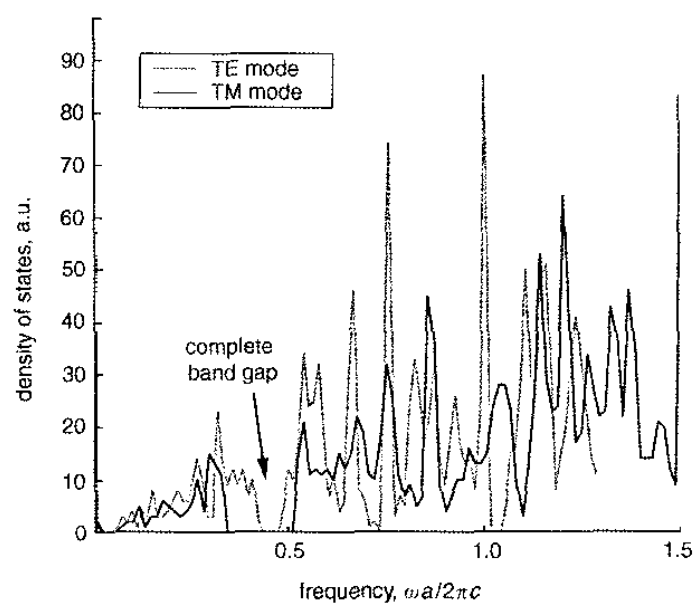

Fig. 5 Density of states diagram for a triangular lattice of air rods in a substrate with dielectric constant of 11.4, filling fraction $=80^{\circ} \%$ The TE and TM modes are represented by the dashed and solid lines respectively. A complete band gap is observed between the normatised frequencies, $\omega a / 2 \pi c=0.44$ and 0.48 .

A FDTD analysis of the same structures [17] shows excellent agreement with this result and the presence of a complete band gap for the triangular lattice with this filling fraction composed of air rods in GaAs has been verified experimentally.
Further verification of the efficacy of the FEM can be found by plotting the eigenvalues as one traverses the irreducible Brillouin zone. This gives the band diagram shown in Fig. $6 a$. This can be directly compared with the equivalent plane wave analysis presented in Fig. $6 b$ [3]. It is clear that the two Figures show excellent agreement.
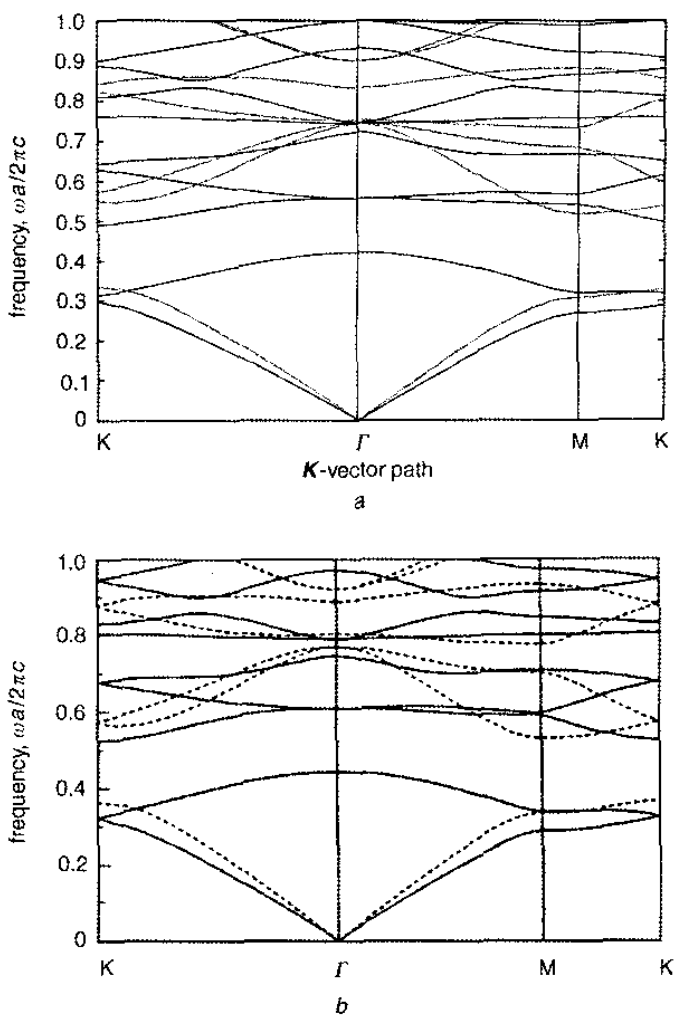

Fig. 6 Band diagrams for a triangular lattice of air rods in GaAs, filling fraction $=80 \%$. The solid and broken curves correspond to the TM and TE modes respectively

$a$ FEM analysis

$b$ Plane wave analysis

Naturally, the crystal geometry affects its optical properties; thus by varying the rod radius ( $r$ ) whilst keeping the pitch (a) constant it is possible to determine where the band gaps exist in the form of a 'gap map' as shown in Fig. 7. Comparison with the equivalent data presented in [17] generated using a plane wave expansion technique again shows excellent agreement.

\section{Algorithm performance}

The relationship between the accuracy of the solution, the mesh size and the interpolation order was investigated using several meshes of varying granularity. These meshes modelled a PC consisting of a triangular lattice of air rods in a substrate material with a dielectric constant of ten and a filling fraction of $50 \%$. The FEM code computed the lowest ten cigenvalues at $76 \boldsymbol{k}$ steps for these meshes using first-to sixth-order interpolation. The computation time and the resulting eigenvalues were recorded. The relative error was computed via comparison to a pseudo-exact solution (10,000 element mesh with fourth-order interpolation).

Plotting computation time against relative error allows the most efficient combination of mesh granularity and interpolation order to be found. These data can be seen 


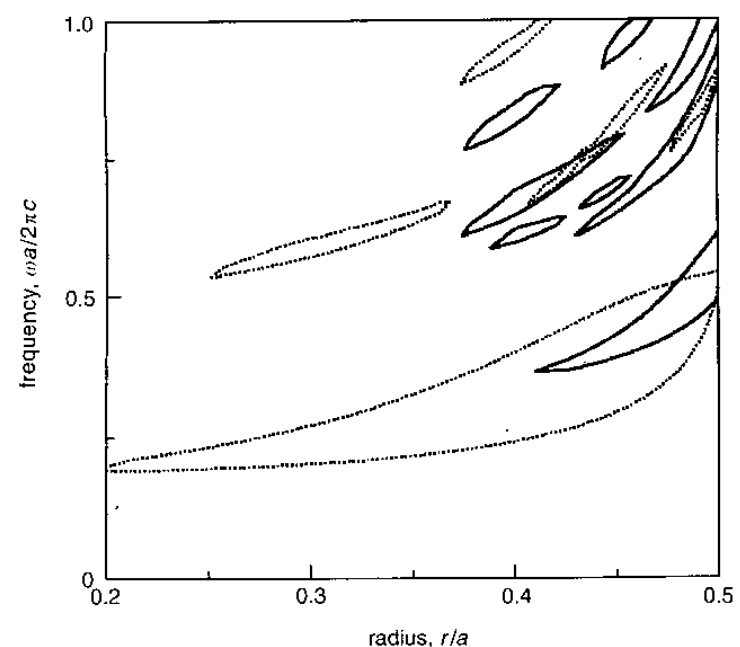

Fig. 7 Gap map for a triangular lattice plotting the band gap frequency ranges for both the TE (dashed) and TM (solid) modes against the rod radius. The data is computed for air rods in GaAs. $A$ complete band gap appears for a rod radius to lattice constant ratio of 0.42 .

in Fig. 8. The clear trend is for the data points to extend from the bottom left-hand corner to the top right-hand corner. This corresponds to coarse meshes producing quick but relatively high error solutions through to fine meshes producing low error solutions more slowly. The ideal

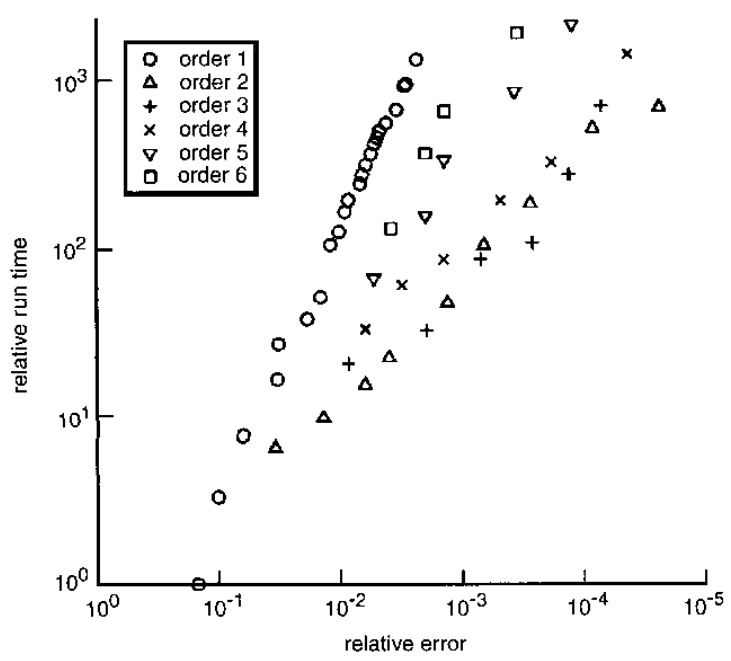

Fig. 8 Solution accuracy as a function of computation time. Meshes of varying granularity were used, and the calculations were performed using a range of interpolation orders.

situation is to compute a solution with low relative error in a short time, i.e. results tending towards the bottom righthand corner of the Figure. Second-and third-order interpolation give the best performance in this respect with orders 4 to 6 becoming worse. First-order interpolation, widely used to date for PC. modelling due to its relatively simple implementation, is significantly worse than all the other interpolation orders. The most accurate first-order calculation took 40 times as long to complete as the secondand third-order calculations yielding similar accuracies.

\section{Conclusions}

Two-dimensional FEM analysis of two-dimensional PCs offers satisfactory agreement with experimental results whilst providing substantial savings in terms of memory and computational costs with respect to fully threedimensional vector FEM implementations. This allows a wide range of crystal structures to be simulated efficiently thus avoiding the necessity of expensive silicon fabrication during the crystal design phase.

The subspace iterative method is very computationally efficient as only a small number of the least dominant eigenvalues need be computed to determine a photonic band gap. This factor coupled with optimisations including preconditioning, exploitation of similarity of adjacent solutions and judicious refinement of the convergence tolerance further increases its efficiency.

Investigation into the performance of the FEM reveals that significant gains in solution quality are made for a given computational cost if second- or third-order interpolation functions are used instead of first order. These are typically speed improvements of at least one order of magnitude.

\section{References}

1 YABLONOVITCH, E.: 'Inhibited Spontaneous Emission in SolidState Physics and Electronics', Phys. Rev. Lett. 1987, 58, (20). pp. $2059-2062$

2 PARKER, G.. and CHARLTON, M.: 'Photonic crystals', Phys. World, 2000, 13, (8), pp. 29-34

3 VILLENEUVE, P.R., and PICHE. M.: Photonic bandgaps in periodic dielectric structures', Prog. Quantum Electron., 1994, 18, (2), pp. 153-200

4 JIN, J.: 'The Finite Element Method in Electromagnetics'. (Wiley, New York. 1993)

5 Niceneco, B., Easymesh quality mesh generator. 2000. http://wwwdinma.univ. Trieste.i1/ nirftc/research/easymesh

6 JOE, B.. Geompack mesh generaing software. 2001. http://www. cs.sunnysb.edu/ $\sim$ algorith $/ \mathrm{implement/geompack/implement.shtml}$

7 KUCHMENT, P.: "Floquet theory for partial differential equations", (Birkhauser Verlag. Basel, Switzerland. 1993)

8 KITTEL, C.: 'Introduction to Solid State Physics', 6th Edn. (John Wiley \& Sons. Inc.. New York. 1986)

9 PRESS W.H. TEUKOLSKY S.A., VETTERLING, W.T., and FLANNERY, B.P.: 'Numerical Recipes in Fortran 77 - The art of scientific computing, (Cambridge University Press. 1999). Vol. 1

10 SEHMI, N.S.: 'Large Order Siructural Eigenanalysis Techniques', (Ellis Horwood, Chichester. 1989)

11 JENNINGS, A.: 'Matrix Computation for Engineers and Scientists'. (Wiley, New York, 1977)

12 BATHE, K.J., and WILSON E L. Numerical Methods in Finite Element Analysis', (Prentice Hall Inc., Princeton, New Jersey. 1976)

13 PISSANETSKY, L.: 'Sparse Matrix Technology', (Academic Press Inc., London. 1984)

14 CHARLTON, M.D.B., ZOOROB, M.E., PARKER, G.J., NETTI, M.C. BAUMBERG, J.J., COX, S., and KEMHADJIAN, $\mathrm{H}$. 'Experimental investigation of photonic crystal waveguide devices and line-defect wavequide bends', Mater. Sci. Eny. B. Solid-State Mater. Adv. Technol., 2000. 74, (1-3). pp. 17-24

15 AXMANN. W. KUCHMENT, P.. and KUNYANSKY. L.: Asymplotic methods for thin high-contrast two-dimensional PBG materials, J. Lightwave Techol., 1999, 17. (11). pp. 1996-2007

16 VILLENEUVE, P.R., and PICHE, M.: 'Photonic Band-Gaps in 2Dimensional Square and Hexagonal Lattices', Phys. Ret. B. Condens. Matter Mater Ply', 1992 46, (8), pp. $4969-4972$

17 JOANNOPOULOS, J.D., MEADE, R.D, and WINN, J.N.: "Photonic crystals: molding the flow of light", (Princeton University Press, Princeton, New Jersey, 1995) 